\title{
Agreement between Direct Fluorescent Microscopy and Ziehl-Neelsen Concentration Techniques in Detection of Pulmonary Tuberculosis in Northwest Ethiopia
}

\author{
Meseret Workineh $^{1 *}$, Mandie Maru ${ }^{2}$, Ibrahim Seman ${ }^{2}$, Ziyadu Bezu ${ }^{2}$, Markos \\ Negash $^{1}$, Mulugeta Melku ${ }^{3}$, Addisu Gize ${ }^{4}$, Agumas Shibabaw ${ }^{5}$
}

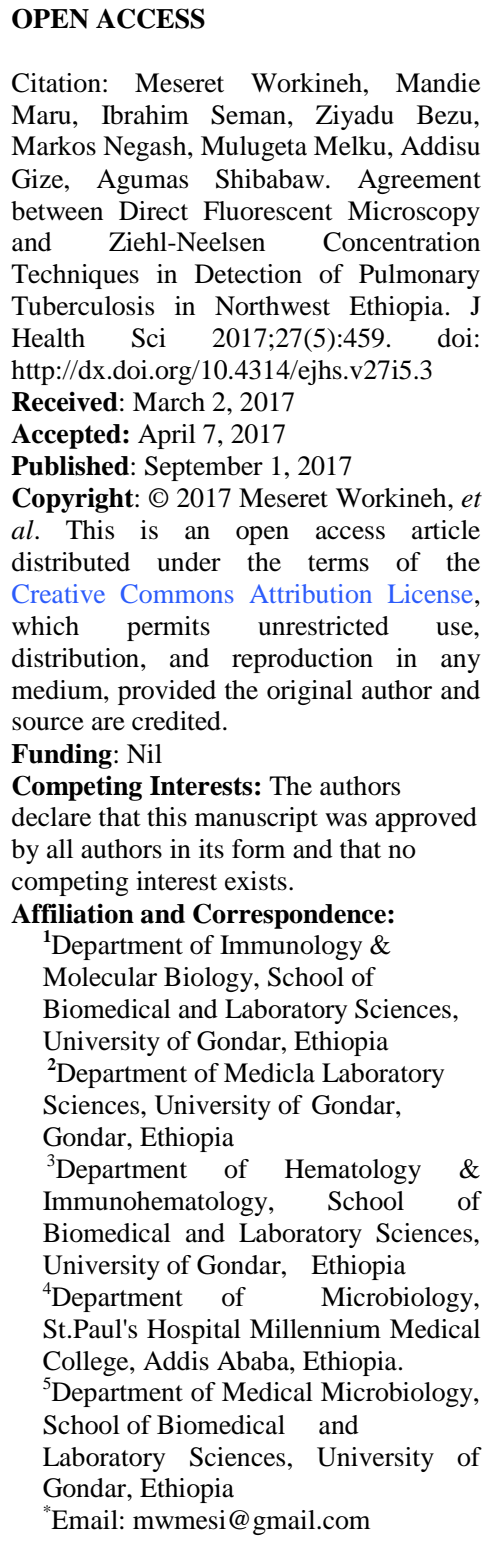

OPEN ACCESS

Citation: Meseret Workineh, Mandie Maru, Ibrahim Seman, Ziyadu Bezu, Markos Negash, Mulugeta Melku, Addisu Gize, Agumas Shibabaw. Agreement between Direct Fluorescent Microscopy and Ziehl-Neelsen Concentration Techniques in Detection of Pulmonary Tuberculosis in Northwest Ethiopia. J Health Sci 2017;27(5):459. doi: http://dx.doi.org/10.4314/ejhs.v27i5.3

Received: March 2, 2017

Accepted: April 7, 2017

Published: September 1, 2017

Copyright: () 2017 Meseret Workineh, et al. This is an open access article distributed under the terms of the Creative Commons Attribution License, which permits unrestricted use, distribution, and reproduction in any medium, provided the original author and source are credited.

Funding: Nil

Competing Interests: The authors

declare that this manuscript was approved

by all authors in its form and that no

competing interest exists.

Affiliation and Correspondence:

${ }^{1}$ Department of Immunology \&

Molecular Biology, School of

Biomedical and Laboratory Sciences,

University of Gondar, Ethiopia

${ }^{2}$ Department of Medicla Laboratory

Sciences, University of Gondar,

Gondar, Ethiopia

${ }^{3}$ Department of Hematology \& Immunohematology, School of Biomedical and Laboratory Sciences, University of Gondar, Ethiopia

${ }^{4}$ Department of Microbiology, St.Paul's Hospital Millennium Medical

College, Addis Ababa, Ethiopia.

${ }^{5}$ Department of Medical Microbiology,

School of Biomedical and

Laboratory Sciences, University of

Gondar, Ethiopia

*Email: mwmesi@gmail.com

\section{ABSTRACT}

BACKGROUND: The sensitivity of smear microscopy for diagnosis of tuberculosis might be improved through treatment of sputum with sodium hypochlorite and application of fluorescent microscopy. This study aimed to determine the agreement between direct Fluorescent Microscopy and Ziehl-Neelsen concentration technique by their ability of detecting acid fast bacilli in resource poor settings.

METHODS: A cross sectional study was conducted at Gondar University Referral Hospital, Northwest Ethiopia. Three sputum specimens were collected from consecutive TB suspects. Direct and concentrated sputum smears were air-dried, heat-fixed and stained by auramine $O$ and Ziehl-Neelsen staining techniques respectively. The stained slides were examined for acid fast bacilli using direct Fluorescent Microscopy and Ziehl-Neelsen concentration techniques.

RESULTS: Of 293 specimens, $4.4 \%$ and $2.4 \%$ were $A F B$ positive by direct fluorescent microscopy and Ziehl-Neelsen bleach concentrated techniques respectively. There was high percentage of tuberculosis positivity from early morning sputum samples (2.4\%) compared to first spot (1.4\%) and second spot (1.7\%) sputum samples when using Ziehl-Neelsen sodium hypochlorite concentration technique. A moderate agreement was seen between the two methods (Kappa $=0.484, P$ value $<0.001)$.

CONCLUSION: Direct fluorescent microscopy has shown high positivity rate compared to Ziehl-Neelsen concentration technique. A moderate agreement was seen between the two methods. Thus, Ziehl-Neelsen bleach sedimentation technique is recommended for detection of pulmonary tuberculosis at peripheral health service level when Fluorescent Microscopy is not available

KEYWORDS: Agreement, Direct Fluorescent Microscopy, ZiehlNeelsen concentration, Tuberculosis, Ethiopia 


\section{INTRODUCTION}

Tuberculosis (TB) is an infectious disease caused by the bacilli belonging to Mycobacterium tuberculosis complex, usually Mycobacterium tuberculosis (1). Tuberculosis remains a major global health problem. According to World Health Oganization (WHO) global tuberculosis report, 9.6 million people were estimated to have fallen ill with TB and TB killed 1.5 million people (1.1 million Human immunodeficiency virus/HIVnegative and 0.4 million HIV-positive) worldwide in 2014 (2). Ethiopia is ranked $7^{\text {th }}$ among the world's 22 high burden countries with an estimated incidence rate of 207 per 100,000 population in 2014 (2).

Early diagnosis of TB is crucial to ensure early identification of cases, and good treatment outcomes. This in turn limits TB transmission, and make TB control strategy effective (3). The diagnosis of pulmonary TB relies heavily on sputum microscopy and culture method. The culture method is the gold standard for pulmonary TB diagnosis. However, due to lack of access to culture facilities and the long turn-around times involved with sputum culture, most laboratories in developing countries where TB is prevalent, utilize direct Ziehl-Neelsen (ZN) microscopy for detection of acid-fast bacilli (AFB) in sputum smears as their main diagnostic tool $(3,4,5)$.

The conventional direct smear microscopy is foremost rapid, inexpensive, highly specific and capable of identifying the most infectious cases of TB. However, the main limitation of the method is its low sensitivity in developing countries, especially in patients co-infected with HIV $(6,7)$. By considering the low sensitivity of direct $\mathrm{ZN}$ microscopy, many studies suggested that the performance of sputum smear microscopy can be significantly improved if sputum is concentrated by centrifugation or sedimentation prior to acidfast staining. Although the resource needed to practice concentrated smearing technique remains a problem in many laboratories, studies suggested that digestion of sputum with household bleach (sodium hypochlorite $(\mathrm{NaOCl})$ ) gives the best recovery of acid-fast bacilli in combination with concentration by centrifugation.
It has also been shown that concentration and liquefaction improve the sensitivity of the AFB smear microscopy and can contribute significantly to achieve better accuracy of diagnosis (8-13).

Recently, fluorescence microscopy is found to be an alternative technique to increase the sensitivity (10\% higher) when compared with $\mathrm{ZN}$ ) microscopy methods while speeding up the whole process to consume much lesser time. Fluorescent acid fast bacilli can be seen at lower magnification, and smears can be examined in a fraction (about 25\% less) of the time than needed for $\mathrm{ZN}$ smears (14).

A systematic review published in 2006 reported that Ziehl-Neelsen could improve sensitivity of direct smear microscopy by $10 \%$ over the conventional $\mathrm{ZN}$ microscopy mostly used in poorer countries, and that specificities of the two techniques were comparable (5). However, the agreement of the fluorescence microscopy with $\mathrm{Zn}$ sodium hypochlorite concentration technique is unclear. Therefore, in this study, we sought to assess the agreement between direct Fluorescent microscopy and $\mathrm{Zn}$ sodium hypochlorite concentration techniques by their ability of detecting acid fast bacilli from pulmonary TB suspected patients.

\section{MATERIALS AND METHODS}

Study setting and design: A cross sectional study was conducted from February to June 2015 at the University of Gondar Referral Hospital (UGRH), Northwest Ethiopia. The hospital has TB clinic where TB patients are get their treatment through the Directly Observed Treatment Short-Course (DOTS) program and further assessment during follow-up period.

Study population: A total of 293 clinically suspected pulmonary tuberculosis patients visiting UGRH TB Clinic participated in the study.

Specimen collection and laboratory processing: Three sputum samples were collected from each patient by using a clean dry and labeled cup after providing instruction on how to produce a good quality sputum according to the WHO and National TB and Leprosy Control Programme recommendation applying the spot-morning-spot approach (15). From all spot-morning-spot

DOI: http://dx.doi.org/10.4314/ejhs.v27i5.3 
specimens, smear were prepared for direct FM technique from good quality sputum, and a small portion of the most suggestive part of the sputum sample was spread using coil type movement $2 \mathrm{~cm}$ by $1 \mathrm{~cm}$ in the center of a new glass slide until a fairly even thickness was obtained. The smear was air-dried, heat-fixed and stained by Auramine O staining technique and examined using fluorescent microscope (FM). The remaining sputum samples were analyzed by $\mathrm{ZN}$ sodium hypochlorite concentration technique by mixing with equal volume of commercially available household bleach $(5 \% \mathrm{NaOCl})$. The mixture was incubated at room temperature for 10-15 minutes, and $8 \mathrm{ml}$ of distilled water was added and centrifuged at 300 rpm for 15-20 minutes and poured off the supernatant and one-two drops from well mixed sediment is transferred with sterile pipette to pre labeled slide. The air-dried and fixed slides were stained by using ziehl-Neelsen staining procedure and observed microscopically for the presence of AFB.

Quality control: The quality of the reagents was checked by staining a known positive smear by following standard operational procedure. Each slide reading for both techniques was performed by two laboratory technologists blindly and discordant result was confirmed by the $3^{\text {rd }}$ laboratory technologist. The result was reported according to WHO/ IUATLD guidelines (16) and graded as negative, scanty and $1+, 2+$ or $3+$ depending on the number of bacilli under the microscope.

Data analysis: Data analysis was performed using SPSS software packages (Version 20.0) after importing the data from Epi Info ${ }^{\mathrm{TM}}$ version 3.5.3. Descriptive statistics was used to show patient socio-demographics characteristics. The chisquare test was used to investigate differences in proportions. A P-value less than 0.05 was considered as statistical significant.

Ethics approval : The study was approved by the ethical committee of School of Biomedical and Laboratory Sciences, University of Gondar. In addition, written informed consent was obtained from each participant.

\section{RESULTS}

A total of two-hundred and ninety-three sputum specimens were collected for the study. Of 293 examined sputum specimens, 7(2.4\%) and 13(4.4\%) were AFB positive by using ZN concentration technique and direct fluorescent microscopy respectively (Table 1 ).

Table 1: AFB detection using Ziehl-Neelsen staining of smears prepared by bleach concentration \& direct fluorescent microscopy.

\begin{tabular}{lll}
\hline Technique & AFB Positive (\%) & AFB Negative (\%) \\
\hline ZN concentration & $7(2.4 \%)$ & $286(97.6 \%)$ \\
Direct FM & $13(4.4 \%)$ & $280(95.6 \%)$ \\
\hline \multicolumn{2}{c}{ Direct FM=Direct fluorescent microscopy, ZN concentration=Ziehl-Neelsen concentration, AFB=Acid fast bacilli }
\end{tabular}

Early morning sputum sample was used for comparison of quantification of $\mathrm{AFB}$ by $\mathrm{ZN}$ concentration technique and direct fluorescent microscopy. The scanty-positive findings are more commonly seen with $\mathrm{ZN}$ sodium hypochlorite concentration technique and not with the direct FM. From a total of 13 positive samples by direct fluorescent microscopy, 8(61.5\%) smears were in the proportion of +1 smear grade. However, more than $70 \%$ of positive specimens were in the proportion of +1 smear grade when using $\mathrm{ZN}$ concentration technique (Table 2).
Although, not statistically significant, there was high percentage of tuberculosis positivity from early morning sputum samples (2.4\%) compared to first spot (1.4\%) and second spot $(1.7 \%)$ sputum samples when using $\mathrm{ZN}$ sodium hypochlorite concentration technique. However, we found almost similar percentage of positivity from early morning, first spot and second spot sputum samples when using direct fluorescent microscopy (Table 3).

DOI: http://dx.doi.org/10.4314/ejhs.v27i5.3 
Table 2: Comparison of quantization of AFB from Ziehl-Neelsen staining of smear prepared by bleach concentration and direct fluorescent microscopy

\begin{tabular}{lll}
\hline Quantification grade & Fluorescent microscopy & ZN concentration \\
\hline Negative & $280(95.6 \%)$ & $286(97.6 \%)$ \\
Total positive & $13(4.4 \%)$ & $7(2.4 \%)$ \\
Scanty & $0(0 \%)$ & $2(28.6 \%)$ \\
$\mathbf{+ 1}$ & $8(61.5 \%)$ & $5(71.4 \%)$ \\
$\mathbf{+ 2}$ & $1(7.7 \%)$ & $0(0 \%)$ \\
$\mathbf{+ 3}$ & $4(30.8 \%)$ & $0(0 \%)$ \\
\hline
\end{tabular}

Table 3: Comparisons of early morning sputum specimen with corresponding spot sputum specimens

\begin{tabular}{|c|c|c|c|c|c|c|}
\hline \multirow[t]{2}{*}{ Sample } & \multicolumn{3}{|c|}{ Fluorescent microscopy } & \multicolumn{3}{|c|}{ ZN concentration } \\
\hline & $\begin{array}{l}\text { Positive } \\
\mathrm{n}(\%)\end{array}$ & $\begin{array}{l}\text { Negative } \\
\mathrm{n}(\%)\end{array}$ & $X^{2}(\mathrm{P}$-value $)$ & $\begin{array}{l}\text { Positive } \\
\mathrm{n}(\%)\end{array}$ & $\begin{array}{l}\text { Negative } \\
\mathrm{n}(\%)\end{array}$ & $X^{2}(\mathrm{P}$-value $)$ \\
\hline $\operatorname{Spot}\left(1^{\text {st }}\right)$ & $12(4.1 \%)$ & $281(95.9 \%)$ & & $4(1.4 \%)$ & $289(98.6 \%)$ & \\
\hline Early morning & $13(4.4 \%)$ & $280(95.6 \%)$ & $0.15(0.92)$ & $7(2.4 \%)$ & $286(97.6 \%)$ & $0.89(0.64)$ \\
\hline $\operatorname{Spot}\left(2^{\text {nd }}\right)$ & $13(4.4 \%)$ & $280(95.6 \%)$ & & $5(1.7 \%)$ & $288(98.3 \%)$ & \\
\hline
\end{tabular}

Among 293 specimens, 278 were found to be negative by both direct FM and $\mathrm{ZN}$ sodium hypochlorite concentration techniques. All the samples which were positive for the $\mathrm{ZN}$ bleach concentration method were positive by the direct fluorescent microscope method also, except two samples. Eight samples were positive by direct florescent microscope alone. These results indicated that there is a moderate agreement between the two methods (kappa value $=0.484, \mathrm{P}$ value $<0.001)($ Table 4$)$

Table 4: Agreement between direct FM and ZN concentration technique among TB suspected patients.

\begin{tabular}{|c|c|c|c|c|c|c|}
\hline \multirow[t]{2}{*}{ Method } & & \multicolumn{2}{|c|}{ Direct FM } & & \multirow[t]{2}{*}{ Kappa value } & \multirow[t]{2}{*}{$P$ value } \\
\hline & & Negative & Positive & Total & & \\
\hline \multirow[t]{2}{*}{ Concentration $\mathrm{ZN}$} & Negative & 278 & 8 & 286 & & \\
\hline & Positive & 2 & 5 & 7 & 0.484 & $<0.001$ \\
\hline Total & & 280 & 13 & 293 & & \\
\hline
\end{tabular}

\section{DISCUSSION}

Improved detection of pulmonary $\mathrm{TB}$ is considered a priority to combat the burden of tuberculosis. The current frontline diagnostic test, smear microscopy, lacks sensitivity. Case finding might be augmented through the implementation of concentration of sputum and applying fluorescence microscopy. This study assessed the agreement between $\mathrm{ZN}$ sodium hypochlorite concentrations and direct Fluorescence Microscopy techniques.

This study revealed that direct fluorescent microscopy showed high positivity rate compared to $\mathrm{ZN}$ sodium hypochlorite concentration technique. Many other studies documented that fluorescent microscopy has been found to be more sensitive than $\mathrm{ZN}$ microscopy (5). In this study, we found eight samples that were positive by direct fluorescent microscopy but negative by concentrated $\mathrm{ZN}$. This may be due to lower

DOI: http://dx.doi.org/10.4314/ejhs.v27i5.3 
specificity of fluorescent microscopy attributable to the appearance of artifacts (17) although it has been found to be more sensitive than $\mathrm{ZN}$ microscopy.

In our study, two sputum specimens that were positive with the $\mathrm{ZN}$ sodium hypochlorite concentration technique were found to be negative by direct fluorescent microscopy. This might have occurred due to inappropriate sample concentration, smear preparation, faulty staining process or inappropriate microscopic observation. Most probably, the saliva part of the sputum might have been taken during smear preparation for detection of AFB by using direct fluorescent microscopy, which might be less likely to occur in $\mathrm{ZN}$ concentration technique because smears were performed after concentrating the sputum by sodium hypochlorite.

Moreover, we found almost similar percentage of positivity from early morning, first spot and second spot sputum samples when using direct fluorescent microscopy. The revised WHO guidelines (18) recommended collecting only two sputum specimens, which would be sufficient and significantly reduce the work load in diagnostic laboratories. As indicated above, our findings also support this idea. There was no difference in three specimens. This is also supported from other studies in Tanzania (19) and Ethiopia (20).

Although, not statistically significant, there was high percentage of tuberculosis positivity from early morning sputum samples compared to the two spot specimens when using concentrated $\mathrm{ZN}$ technique. In line with the findings of our study, a study from Zambia also showed that more than $93 \%$ of TB cases were diagnosed by early morning specimens. Consequently, many researchers have suggested using only two sputum specimens as the basis of case finding which would reduce the number of visits required for diagnosis and save resources for the health system and the patients (21).

The scanty-positive findings are more commonly seen with $\mathrm{ZN}$ concentration technique and not with the direct FM. This could be attributed to the low-power objective of the fluorescence techniques that allow the field to be seen larger than in the conventional $\mathrm{ZN}$ light microscopy. Some of the samples studied may have come from HIV co-infected patients with low-density bacilli yielding scanty $\mathrm{AFBs}$ on the concentrated $\mathrm{ZN}$ technique. On the contrary, grades of $2+$ and $3+$ findings were commonly seen with direct FM than concentrated $\mathrm{ZN}$ technique. However, the fluorescence techniques showed the highest numbers of false positives compared with the $\mathrm{ZN}$ light microscopy. This may be due to the use of low magnification in the fluorescence microscopy might increase the proportion of naturally fluorescent artifacts such as fungi spores to be seen as AFB positive. $(22,23)$.

Many studies have showed that concentrated $\mathrm{ZN}$ technique has better sensitivity than direct microscopy methods. However, there are some problems with its feasibility in resource poor settings where tuberculosis is highly prevalent. Limited human and financial resources, inadequate training capacity, lack of proper biosafety arrangements, and potential biohazard posed by centrifugation are some of the feasibility problems in $\mathrm{ZN}$ concentration technique. In this case, although it has some disadvantages, direct FM method can be alternatively applied for laboratory diagnosis in these resource poor settings. Our findings revealed that there is a moderate agreement between these two methods.

\section{ACKNOWLEDGEMENTS}

We would like to thank University of Gondar, School of Biomedical and Laboratory Sciences for its funding. We also extend our acknowledgment to the study participants without whom this work would have not been a reality. We also acknowledge the nurses and laboratory technicians of DOTS Clinic at University of Gondar Referral Hospital, who screened and recruited patients for the study and proced sample.

\section{REFERENCES}

1. Kumar V, Abbas AK, Fausto N, Mitchell RN. Robbins Basic Pathology, $8^{\text {th }}$ ed. Saunders Elsevier, PP. 516-522, 2007. 
2. World Health Organization (WHO), Global Tuberculosis Report, WHO; 2015.

3. Huebner RE, Good RC, Tokars JI. Current practices in mycobacteriology: results of a survey of state public health laboratories. J Clin Microbiol. 1993; 31(4):771775 .

4. Aber VR, Allen BW, Mitchison DA, Ayuma P, Edwards EA, Keyes AB. Quality control in tuberculosis bacteriology. Laboratory studies on isolated positive cultures and the efficiency of direct smear examination. Tubercle. 1980; 61(3):123-133.

5. Steingart KR, Henry M, Ng V, et al. Fluorescence versus conventional sputum smear microscopy for tuberculosis: a systematic review. Lancet Infect Dis.2006;6(9):570-581.

6. Getahun H, Harrington M, O'Brien R, Nunn P. Diagnosis of smear-negative pulmonary tuberculosis in people with HIV infection or AIDS in resource constrained settings: informing urgent policy changes. Lancet. 2007;369(9578):2042-2049.

7. Corbett EL, Watt CJ, Walker N, et al. The growing burden of tuberculosis: global trends and interactions with the HIV epidemic. Arch Intern Med. 2003;163(9):1009-1021.

8. C. Habeenzu. D, Lubasi. A. Fleming, Improved sensitivity of direct microscopy for detection of acid fast bacilli in sputum in developing countries. Trans. R. Soc. Trop. Med. Hyg. 1998;92(4): 415416.

9. H. Miorner, G. Gunilla, Y. Zemene, A. Yengusnesh. Improved sensitivity for acid-fast Bacilli: Sedimentation as an alternative to centrifugation for concentration of tubercle bacilli. J Clin Microbiol. 1996;34(12):3206-3207.

10. Steingart $\mathrm{KR}, \mathrm{Ng} \mathrm{V}$, Henry $\mathrm{M}$, et al. Sputum processing methods to improve the sensitivity of smear microscopy for tuberculosis: a systematic review. Lancet Infect Dis. 2006;6(10):664-674.

11. Cattamanchi A, Davis JL, Pai M, et al. Does bleach processing increase the accuracy of sputum smear microscopy for diagnosing pulmonary tuberculosis. J Clin Microb.2010;48(7):24332439.

12. Bonnet $\mathrm{M}$, Gagnidze L, Githui $\mathrm{W}$, et al. Performance of LED-based fluorescence microscopy to diagnose tuberculosis in a peripheral health centre in Nairobi. PLOS ONE. 2011; 6: e17214.

13. Anagaw B, Mulu A, Abate E, et al. Improved detection of acid fast bacilli by the bleach concentration technique at the Gondar university teaching hospital. Ethiop Med J. 2012;50(4):34954.

14. Armstrong D. LeD-based fluoroscopy and the Para Lens system: illuminating the future of tuberculosis diagnostics. Tuberculosis.2009; 7:1718.

15. World Health Organization. Treatment of Tuberculosis: guidelines for national programmes. 2nd ed. WHO/TB/97.220. Geneva, Switzerland: WHO; 1997.

16. World Health Organization. Laboratory services in tuberculosis control. WHO/TB/98.258. Geneva: WHO; 1998.

17. Schoch OD, Rieder P, Tueller C, et al. Diagnostic yield of sputum, induced sputum, and bronchoscopy after radiologic tuberculosis screening. Am $J$ Respir Crit Care Med. 2007; 175:80-6.

18. World Health Organization, Treatment of Tuberculosis: Guidelines for national programmes, $4^{\text {th }}$ edition, WHO/TB/97.220. Geneva, Switzerland: WHO; 1997.

19. Ipuge YA, Rieder HL, Enarson DA. The yield of acid-fast bacilli from serial smears in routine microscopy laboratories in rural Tanzania.Trans Roy Soc Trop Med Hyg. 1996;90:285-261.

20. Cambanis A, Yassin M A, Ramsay A, Squire SB, Arbide I, Cuevas LE. A one-day method for the diagnosis of pulmonary tuberculosis in rural Ethiopia. Int J Tuberc Lungs Dis. 2005;10(2):230232.

21. Walker D, McNerney R, Mwembo MK, et al. An incremental cost-effectiveness analysis of the first, second and third sputum examination in the diagnosis of pulmonary tuberculosis. Int J Tuberc Lung Dis.2000;4:246-251.

22. Cattamanchi A, Davis JL, Worodria W, et al.Sensitivity and specificity of fluorescence microscopy for diagnosing pulmonary tuberculosis in a high HIV prevalence setting. Int J Tuberc Lung Dis, 2009;13:1130-1136.

23. Chaidir L, Parwati I, Annisa J, et al. Implementation of LED fluorescence microscopy for diagnosis of pulmonary and HIV-associated tuberculosis in a hospital setting in Indonesia. Plos One.2013;8:e61727. 